\title{
Mining Blocks' Association Rules for Disk Data Perfecting
}

\author{
L.Y. Zhu, G.Q. Xiao*, J.W. Liao \\ College of Computer and Information Science \\ Southwest University Chongqing \\ China \\ *Corresponding author
}

\begin{abstract}
I/O gap continues widening, the modern I/O-bound applications such as Online Transaction Processing (OLTP) often suffer from great latency of disk access. In this paper, we argue that the block association rule can be recognized as common semantic information embedded in disk I/O traces and it can be utilized to direct disk prefetching. We propose a novel mining approach to extract such association rules to benefit prefetching. The experimental results show that this proposed approach is effective in increasing prefetching accuracy, and the disk latency can be reduced by this association rule based prefetching.
\end{abstract}

Keywords-association rule; prefetching; storage system

\section{INTRODUCTION}

As the speed gap between processor and disk continues to grow, the disk-based storage system is becoming the bottleneck of data-intensive application system. We are now allowed to apply much more delicate methods to the storage system to attack the problem. Prefetching is a common solution to mask the I/O latency. This approach hides the latency with early $\mathrm{I} / \mathrm{O}$ initiation by utilizing the relatively redundant computational resource, as well as efficient and accurate prediction algorith $\mathrm{m}$.

In general, there are mainly two fundamental perfecting techniques [1] [2]: heuristic and speculative schemes. The later approach 'pre-executes' fragments of code in the targeted process via an extra helper thread [3] [4] [5]. The speculative execution thread only keeps track of I/O related operations and identifies future $\mathrm{I} / \mathrm{O}$ references. This kind of approach generally achieves high accuracy and can be more suitable for applications whose access patterns are complex or random. However, it usually involves source code transformation, prefetching injection and hardware support. On the other hand, the heuristic scheme predicts future access based on patterns obtained by analyzing past access history (or so-called trace) [5] [6] [7]. This approach can be implemented without hardware support, and has no need to touch the application.

In response to all these observations, we propose a novel association rule mining approach used for heuristic prefetching scheme. It is designed for improving the $\mathrm{I} / \mathrm{O}$ performance for complex workloads. Our mining approach solely targeting at conducting prefetcher, outputs the association rules that can be directly used to predict future data access. The mining approach is optimized in order to extract a s mall number of necessary rules.

\section{EXPLOITING BLOCK CORRELATIONS AS SEMANTICS}

\section{A. Block Correlations}

The essential idea of block correlations is based on the fact that the data processed and stored are somehow related with each other by the semantics they contain. Two blocks can be considered 'linked' if the data stored by these blocks is correlated. However, data semantics can be much more complex and rich, thus making the block correlations hard to detect. Consider the correlation involves blocks that store indexes and data the indexes point to in database systems. It is both depended on high-level semantics of the data itself and the data structures the application defines. Therefore it is difficult to derive a general yet effective mathematical description of these kinds of correlations.

Association rule [10] is a straightforward expression of block correlation and can be directly used to predict future block reference. These rules describe a phenomenon that certain items occur with a high frequency after an occurrence of other specific bunch of items. If we discover that block 5 is always requested following block 1, 2 and 3, then we obtain one association rule represented as $\{1,2,3\}->\{5\}$, where the left hand $\{1,2,3\}$ is called antecedent and right hand is called consequent. We only need to mine one-item-consequent rules here.

\section{B. Time Constrains}

In order to constrain the search space, time constrains are applied to the association rules. It is obvious that those rules are of less use even when they are indeed frequent and highly confident, if whose antecedents have a long time span or there is a huge time lag between the two antecedents and consequents.

Formally, a time window $\mathrm{w}$ on a block sequences is represent as $w[\mathrm{Ts}, \mathrm{Te}]$, where $T s$ and $T e$ are start time and end time of window w,. Usually we constrain the width of the searching window so that only closely occurring sequences are searched. Besides, the consequent is required to occur in a limit period of time right after antecedent stops, which means if the antecedent stops at time $\mathrm{t} 1$, and the maximum time lag is defined as $t l$, then the consequent should occur within the window of $[t 1, t 1+t l]$. 


\section{IMPLEMENTATION OF MINING ANDPREDICTION}

\section{A. Preprocessing}

There are three tasks for this stage. First, it is required that block references are indexed in a single unified-space, which means we can only access only one disk. However, many common publicly available disk traces consist of an array of multiple disks. Each block reference in these traces is indexed by two variables, disk number and block number in that disk. Thus, a transformation is needed to map the two dimensional indexing space to the unified one dimensional space.

Second, there may be two or more blocks occurring at the same timestamp in some traces. At this stage, the timestamp of one of the blocks with identical timestamp is added with a tiny value to differ from another one.

Third, the orig inal long trace is cut up into smaller pieces in fixed length. And each small piece of trace is one by one mined in the first mining stage.

\section{B. Mining all One-Item-Antecedent Rules}

The first mining procedure is to extracting one-item antecedent rules. The algorith $m$ repeatedly $s$ cans each piece, records all qualified rules for each scan, and then merge the rules with those recorded in the previous scans. The following pseudo-code shows the algorithm.

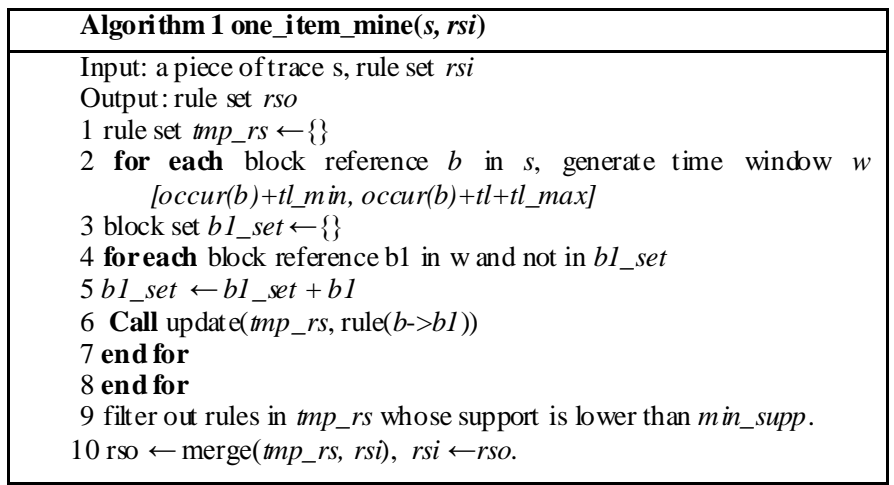

The denotation occur(b) in line 2 represents the timestamp of block $b$, and update in line 6 adds a new rule $b->b 1$ to tmp_rs if this rule is not found in tmp_rs, or updates rule's support and confidence if it is already in tmp_rs. Ideally, if we run the algorithm using the whole trace as input, we will not fail to count those rules that happen to span across two pieces. Due to limited capacity of memory, we have to apply divide and conquer approach. According to [7], the possibility of an instance of a frequent rule span over the cutting point is small, so the inherent loss is acceptable.

\section{Expending Antecedent}

Initially, the algorithm takes one rule extracted by last stage as input, then recursively expands antecedent by one item each time, and stops on some termination criteria. In fact, once one-item-antecedent rule set is obtained, prefetcher is able to work using these rules. However, the expansion stage is necessary. This is because of the observation that there are many rules sharing the same antecedent. In response to this issue, antecedent-expansion further mines the pattern, clears out the ambiguity of shorter rules, and directs the prefetcher making more confident and precise decisions. However, expansion is not always necessary when rules are already highly confident. So we skip the rules with confidence above 0.9 .

The expending algorith $\mathrm{m}$ is as follows:

\section{Algorithm 2 expand $(r)$}

\section{Input: rule $r$ to be expanded}

Output: rule set $r s$, return 1 , if rule $r$ or one of more of its descendants saved. Return 0 otherwise.

1 rule set $t m p \_r s \leftarrow\{\}$

2 for each instance $i$ of $r$

$3 t s$, te refer to timestamp of antecedent's start/stop, and $t c$ is timestamp of consequent's it em; searching window $w[t e-t w, \min (t s+t w, t c)]$

4 for each block $b$ in $w$, such that $b>\max \_$bnr $(r)$ and $\operatorname{rule}(b->\operatorname{cons}(r))$ is included in rule set produced by last stage

$5 \quad$ Call update $\left(t m p \_r s, \operatorname{rule}(\{\operatorname{ante}(r), b\}->\operatorname{cons}(r))\right.$

6 end for

7 end for

8 flag $\leftarrow 0$

9 for each candidate rule $r$ in tmp_rs such that $\operatorname{supp}(r c)>m$ in_support

10 if $\operatorname{conf}(r c)>$ cutoff_conf put $r c$ into $r s$ and flag $\leftarrow 1$

12 else if $\operatorname{conf}(r c)>\operatorname{conf}(r)$ and expand $(r c)==1$

13 flag $\leftarrow 1$

14 end if

15 end for

16 if flag $==0$

17 if conf $(r)>m$ in_conf

18 put $r$ into $r s$ and flag $\leftarrow 1$

19 end if

20 endif

21 return flag

Function update () does the same task as the one in previous stage and conf(r) denotes the confidence of rule $r$. The variable tw is pre-defined maximum searching time windows described in section 2.3. This algorithm consists of two parts. The first part collects all possible candidate blocks in a limited search space. Notice line 9, It only picks up blocks with greater numbers than those already included in rule r's antecedent so that it is guaranteed that no duplicate blocks are brought in. The second constrain is based on following lemma: if rule $\{a\}->\{b\}$ is infrequent, none of the rules whose antecedents consists of item a and share the same consequent are frequent.

In the second part, the algorithm decides whether continue the searching and whether save current rule r. Only the candidates rules that have higher confidence than their ascendant but not higher than cutoff_conf appears in line 10 should be worth further mining, so that all rules left in rule set rs are at their highest confidence and searching space is pruned as another benefit.

\section{EVALUATION}

\section{A. Methodology}

To evaluate the performance of our approach, a tracedriven simulator is implemented. It consists of a cache, interfaces of replacement policy, prefetcher and DiskSim. When the simulator reading the disk trace, it maintains the cache and provokes replacement policy and prefetcher. The actual disk access generated by the simulator is forwarded to DiskSim [11], which simulates accesses being handled by real disk system. 
We select the following traces for our experiments:

1) TPC-C trace is collected on a storage system backing a Microsoft SQL Server. The trace is generated during the clients running TPC-C benchmark for 2 hours. 2) OLTP trace is offered by Storage Performance Council. The trace samples 1.5 hours of I/O activity of an OLTP application running at a financial institution. The storage system is composed of 19 disks. 3) Kernel trace is collected by the authors during compiling Linux kernel. The compiling computer runs a modified Linux kernel, which intercepts VFS's routines and records every disk block references. The total time is about 48 minutes.

We only use the first half part of trace to mine association rules. And evaluate the performance of prefetcher directed by the rules using the second part. We also implement sequential prefetching algorithm based on the same idea adapted by readahead [8] in Linux kernel. All experiments use Least Recent Used (LRU) cache replacement policy.

\section{B. Constrains' Impact on Performance}

Figure 5-1 and 5-2 show the effects of hit ratio by two constrains, minimu m confidence min_conf and maximu $\mathrm{m}$ time searching window tw. To evaluate the performance of our approach, we measure the response time produced by the simulators without prefetching and with sequential prefetching, and figure 5-3 compares the results.

When min_conf decreases, more association rules satisfy the criteria. We can see the ratio increases slowly when confidence is small and finally stop rising at some point. This is because when the criterion is low, too many low quality rules are kept. And as it goes higher than certain points, although the prefetched blocks are highly possible being hit, the chance of triggering prefetching becomes rare and stabilized.

Constrain tw denotes the maximum time window allowed, which limits the searching space and the number of items of antecedents. As figure 1 and 2 show, although all traces share the same tendency that wider the windows lead to higher the hit ratio, the hit ratios themselves respond to tw differently. There are steep rise and stabilizing two stages for TPC-C and OLTP hit ratio, while in kernel trace it increases steadily. W ith this prior knowledge of the targeted workload, we can tune the mining algorith $\mathrm{m}$ faster.

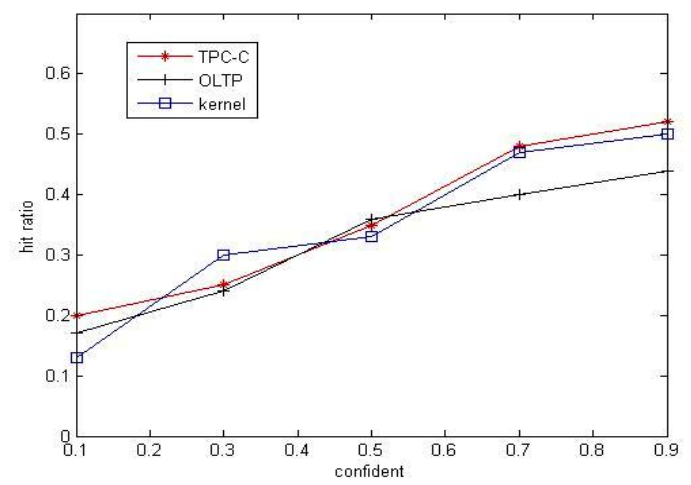

FIGURE I. HIT RATIO AGAINST CONF.

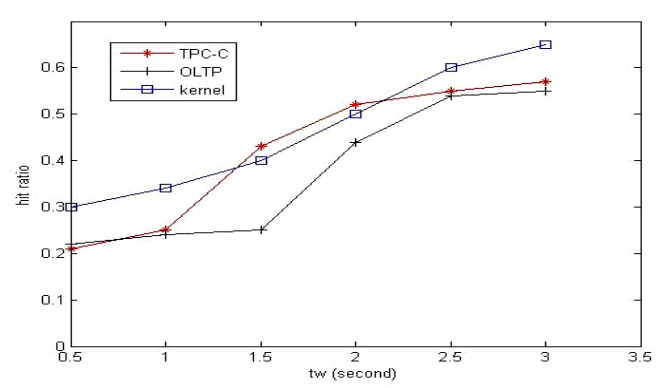

FIGURE II. HIT RATIO AGAINST SEARCHING WINDOW.

Figure 3 shows response times produced by the three traces. In each bar group, the left bar is simulated with none prefetcher, middle bar is with sequential prefetcher, and right one is with our association rule guided prefetcher.

Our prefetcher can effectively decrease the response time over these three traces, especially on TPC-C and OLTP, considering the measurements decrease by $8 \%-10 \%$ comparing with the experiments with none prefetching. We observe that when simulating using sequential policy on TPC$\mathrm{C}$ trace, the response time even goes higher than the one with none prefetcher. Although its performance can be promoted by tuning the policy, the response time cannot decrease as much as our approach achieves.

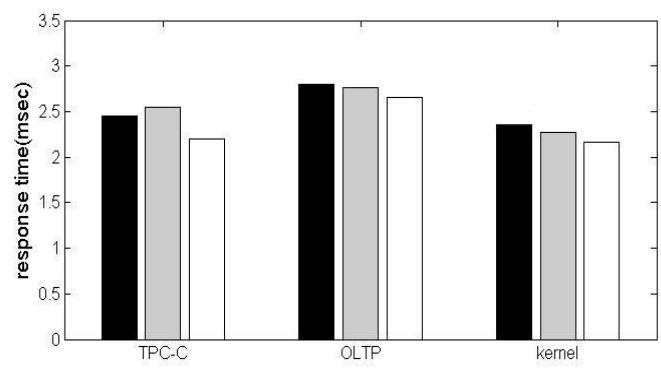

FIGURE III. RESPONSE TIME BYT HREE CONFIGURATIONS, IN EACH GROUP, LEFT BAR IS SIMULATION WITH NONE PREFET CHER, MIDDLE BAR IS WITH SEQUENTIAL PREFET CHER AND RIGHT ONE IS WIT H OUR ASSOCIATION RULE GUIDED PREFETCHER.

\section{CONCLUSION}

In this paper, we have proposed an association rule mining approach designed for the prediction purpose for storage systems. We have discussed the idea of using the correlation information of $\mathrm{I} / \mathrm{O}$ access events in the block-level to direct the prefetcher operating for complex workloads. Therefore, an algorithm for extracting association rule is proposed to contribute to prefetching. The evaluation experiments on real workloads have shown that our approach can efficiently discover a set of accurate prediction ru les at an acceptable cost, as a consequence, the performance of the storage system can be promoted by the association rule based prefetching.

\section{ACKNOWLEDGEMENT}

In this paper, the research was sponsored by National Key Technologies Research and Development Program of China (Project No. 2013BAD15B06). 


\section{REFERENCE}

[1] Zhen, Yong, et al. "Hiding I/O latency with pre-execution prefetching for parallel applications." High Performance Computing, Networking, Storage and Analysis, 2008. SC 2008. International Conference for. IEEE, 2008.

[2] Chang, Fay W. Using speculative execution to automatically hide I/O latency. No. CMU-CS-01-172. CARNEGIE-MELLON UNIV PITT SBURGH PA SCHOOL OF COMPUTER SCIENCE, 2001.

[3] Liao, Jianwei, et al. "Dynamic Stripe Management Mechanism in Distributed File Systems." Network and Parallel Computing. Springer Berlin Heidelberg, 2014. 497-509.

[4] Yang, Chuan-Kai, Tulika Mitra, and Tzi-cker Chiueh. "A Decoupled Architecture for Application-Specific File Prefetching." USENIX Annual Technical Conference, FREENIX Track. 2002.

[5] Byna, Surendra, et al. "Parallel I/O prefetching using MPI file caching and I/O signat ures." High Performance Comput ing, Net working, Storage and Analysis, 2008. SC 2008. Internat ional Conference for. IEEE, 2008.

[6] Jiang, Song, et al. "A Prefetching Scheme Exploiting both Data Layout and Access History on Disk." ACM Transactions on Storage (TOS) 9.3 (2013): 10.

[7] Li, Zhenmin, et al. "C-Miner: Mining Block Correlations in Storage Systems."FAST. 2004.

[8] Fengguang, W. U., X. I. Hongsheng, and X. U. Chenfeng. "On the design of a new Linux readahead framework." ACM SIGOPS Operating Systems Review42.5 (2008): 75-84.

[9] Yan, Xifeng, Jiawei Han, and Ramin Afshar. "CloSpan: Mining closed sequential patterns in large datasets." Proceedings of SIAM International Conference on Data Mining. 2003.

[10] Kotsiantis, Sotiris, and Dimitris Kanellopoulos. "Association rules mining: A recent overview." GEST S International Transactions on Computer Science and Engineering 32.1 (2006): 71-82.

[11] Ganger, Greg, B. Worthington, and Y. Patt. "The DiskSim simulation environment (v4. 0)." (2009). 